\section{MAGNETIC RESONANCE VELOCITY VECTOR MAPPING OF BLOOD FLOW IN THORACIC AORTIC ANEURYSMS AND GRAFTS}

Magnetic resonance imaging with multidirectional cine velocity mapping was used to study relationships between aortic blood flow patterns and the geometry of thoracic aortic aneurysms and grafts. Ten patients with $\mathbf{1 3}$ thoracic aortic aneurysms, single or multiple, or grafts (4) participated in the study. The causes of disease were atherosclerosis (4), Marfan's syndrome (2), trauma (1), and unknown (1), and there were two dissections. Spin-echo imaging and cine velocity mapping in $10 \mathrm{~mm}$ thick slices with vertical and horizontal velocity encoding were done. Maps of the two velocity components were processed into multiple computer-generated streaks whose orientation and length corresponded to velocity vectors in the chosen plane. The dynamic arrow maps were compared with previously reported aortic arrow maps from normal subjects. The forward flow occupied the entire lumen in the normal aorta in systole and small vortices were only present in the sinuses of Valsalva. Atherosclerotic aneurysms in the ascending aorta were located at the anterior right and had oblique, eccentric jet flows that created a large secondary vortex in the aneurysm. Patients with Marfan's syndrome had a central jet and two large vortices, one on each side. All other aneurysms, dissections, and grafts had irregular flows and vortices not seen in normal subjects. Magnetic resonance imaging with multidirectional velocity mapping is a powerful noninvasive tool to assess morphologic features and disturbed blood flow in aortic aneurysms and grafts. Recognizably altered flow patterns were found to be associated with altered vessel geometry. The significance of this requires further investigation. (J THORAC Cardiovasc SuRg 1995;110:704-14)

Hugo G. Bogren, MD, PhD,* Raad H. Mohiaddin, MD,

Guang Z. Yang, PhD, Philip J. Kilner, MD, and David N. Firmin, PhD, London, United Kingdom
$T^{n}$ he role of hydraulic forces in the formation and propagation of aortic aneurysms is still poorly understood. The initial change in the formation of an aneurysm is structural and results from a degen-

From the Royal Brompton Hospital, National Heart and Lung Institute, London, United Kingdom.

Supported by CORDA (Coronary Artery Disease Research Association, London) and by the University of California at Davis.

Received for publication June 13, 1994.

Accepted for publication Dec. 8, 1994.

Address for reprints: Hugo G. Bogren, MD, Department of Radiology, UC Davis Medical Center, 2516 Stockton Blvd., Sacramento, CA 95817.

"On sabbatical leave from the Department of Radiology, University of California, Davis, Medical Center, Sacramento, Calif.

Copyright (C) 1995 by Mosby-Year Book, Inc.

$0022-5223 / 95 \$ 5.00+0 \quad \mathbf{1 2 / 1 / 6 2 9 4 2}$ erative process in the vascular wall. Once the morphologic change has occurred the blood flow pattern is likely to alter, which may cause altered shear stresses and consequent strains and accelerate the propagation of the aneurysm so that a vicious circle is created. The geometry of the aorta is altered in aneurysmal degeneration, and the geometric relationship between the left ventricle and the aortic valve on the one hand and the ascending aorta on the other becomes more and more distorted during the propagation of an ascending aortic aneurysm. Similar morphologic changes occur in aneurysms in the aortic arch or descending aorta that are likely to influence blood flow patterns and change the hydrodynamic forces. The interplay and counterplay between morphologic changes and hydraulic dynamic forces in the development of aortic dissections are also unknown and increased knowledge about the blood flow patterns in dissections might increase our understanding of the pathophysiologic features of 
Table I. Patient data

\begin{tabular}{|c|c|c|c|c|c|c|c|c|}
\hline No. & Age/sex & Diagnosis & $\begin{array}{l}\text { Degree of } A R \text { on } \\
\text { scale from } 1 \text { to } 4\end{array}$ & Pathologic type & $\begin{array}{l}\text { Angle } \\
\text { aorta }\end{array}$ & $\begin{array}{c}\text { Angle } \\
\text { LV }\end{array}$ & $\begin{array}{c}\text { Aortal } \\
\text { LV }\end{array}$ & $\begin{array}{l}\text { An/aorta } \\
\text { width }\end{array}$ \\
\hline 1 & $20 / \mathrm{M}$ & $\mathrm{AA}, \mathrm{An}$ & $2 / 4$ & Marfan's syndrome & 70 & 45 & 25 & $66 / 26=2.5$ \\
\hline 2 & $59 / \mathrm{F}$ & $\mathrm{AA}, \mathrm{An}$ & $2 / 4$ & Marfan's syndrome & 77 & 42 & 35 & $65 / 25=2.6$ \\
\hline 3 & $63 / \mathrm{F}$ & $\mathrm{AA}, \mathrm{An}$ & $1-2 / 4$ & Atherosclerosis & 50 & 27 & 23 & $70 / 24=3.2$ \\
\hline 4 & $70 / \mathrm{M}$ & $\mathrm{AA}, \mathrm{An}, \mathrm{DA}$, dissection & - & Atherosclerosis & 100 & 35 & 65 & $56 / 17=3.4$ \\
\hline 5 & $77 / \mathrm{F}$ & $\mathrm{AA}, \mathrm{An}$ & $2 / 4$ & Atherosclerosis & 87 & 40 & 47 & $81 / 22=3.7$ \\
\hline 6 & $39 / \mathrm{M}$ & AA, An & - & Unknown & 35 & 26 & 9 & $60 / 25=2.4$ \\
\hline 7 & $62 / \mathrm{F}$ & Graft $\mathrm{AA}$, dissection DA & $1-2 / 4$ & Dissection & 60 & 46 & 14 & $42 / 17=3.0$ \\
\hline 8 & $79 / \mathrm{M}$ & Graft AA, dissection arch-DA & - & Dissection & 53 & 29 & 24 & $38 / 23=1.3$ \\
\hline 9 & $62 / \mathrm{F}$ & Graft arch & - & Atherosclerosis & 30 & 0 & 30 & $44 / 19=2.3$ \\
\hline 10 & $30 / \mathrm{F}$ & Graft DA & - & Trauma & 68 & 42 & 26 & $28 / 17=1.7$ \\
\hline
\end{tabular}

Angle aorta and LV denotes the angle (degrees) between the transverse plane and the longitudinal axis of the aorta and the left ventricle. Aorta/LV is the angle between the longitudinal axes of the aorta and LV. An/Ao width denotes the ratio between the width of the aortic valvular ostium in millimeters and the maximal measured width of the aneurysm. $A R$, Aortic regurgitation; $L V$, left ventricle; $M$, male; $A n$, aneurysm; $A A$, Ascending aorta; $F$, female; $D A$, descending aorta.

the disease process. The final event in the natural history of aneurysms is that they rupture and cause death or that they are surgically replaced with a graft. In this latter case, what are the blood flow patterns in surgical grafts? Do they restore normal blood flow patterns or do they create their own hydrodynamic problems?

A noninvasive tool to visualize blood flow patterns by vector analysis of multidirectional cine magnetic resonance velocity mapping was described by members of our research group and others. ${ }^{1-6}$ Mohiaddin and associates ${ }^{7}$ described the technique to visualize aortic and pulmonary aneurysms and the reader is referred to that article and the other referenced articles for technical details about the method.

The aim of the present study was to expand the analysis of blood flow patterns in thoracic aortic aneurysms and grafts to try to answer some of the preceding questions, that is, those concerning the interplay or counterplay, or both, between morphologic features and hydraulic hydrodynamic forces in the formation and propagation of aneurysms in the thoracic aorta.

\section{Material and methods}

Subjects. Ten patients, aged 20 to 79 years (average 56 years), with 13 thoracic aneurysms or grafts ( 3 patients had two aneurysms) were studied in the supine position at rest with spin-echo imaging and velocity mapping. The causes of the aneurysms were atherosclerosis (4), Marfan's syndrome (2), dissection (2), trauma (1), and unknown (1), and there were four patients with grafts. Five patients had mild or mild to moderate aortic valvular regurgitation (Table I).
Four more patients with ascending aortic aneurysms, two with atherosclerosis and two with Marfan's syndrome, underwent study with spin-echo imaging only.

The data from the patients were compared with those published earlier from 10 normal subjects. ${ }^{8}$ These 10 subjects had velocity maps done of the aortic arch and the adjacent parts of the ascending and descending aorta, and three of them had maps done of the proximal ascending aorta as well.

The studies on the patients and the normal subjects were approved by the Ethical Committee of the Royal Brompton Hospital and all subjects signed informed consent forms.

Magnetic resonance imaging (MRI). A Picker International Vista magnetic resonance machine (Picker International, Inc., Healthcare Products Div., Mayfield Village, Ohio) operating at 0.5 tesla with modified gradient coils and a surface receiver coil was used. Cardiac gated transaxial and coronal multislice spin-echo images (echo time $40 \mathrm{msec}$ ) were acquired covering the heart and the thoracic aorta. This was followed by an oblique coronal image equivalent to a left anterior oblique image in the plane of the thoracic aorta located by three points: one at the proximal ascending aorta, one at the top of the aortic arch, and one in the descending aorta. This plane was varied somewhat depending on the location of the examined aneurysm (ascending or descending aorta or the arch). Sometimes a coronal plane was used if such a plane turned out to be the optimal one for visualization of the abnormality in the thoracic aorta. These planes, in most cases a plane aligned with the aortic arch including the aneurysm or graft, was then used for bidirectional cine velocity mapping with a gradient echo sequence with an echo time of $12 \mathrm{msec}$. Sixteen electrocardiogram-gated frames through the cardiac cycle were acquired and the sequence was run three times, once without velocity encoding and two times with velocity encoded in the direction of the read (vertical in the figures) and phase (horizontal) gradient directions. Slice thickness was 10 $\mathrm{mm}$ and the field of view $40 \mathrm{~cm}$. The images were 


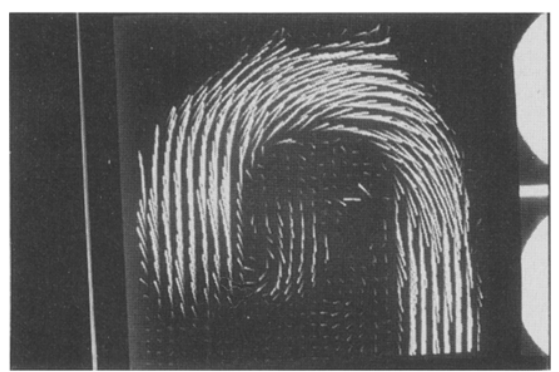

Fig. 1. Image from normal subject with regular aortic systolic flow without vortices.

displayed on a $256 \times 256$ matrix from data acquired from two averages of 128 phase-encoding steps. The acquisition of the bidirectional cine maps took 12 to 15 minutes depending on heart rate, and the whole procedure took approximately 1 hour.

Flow visualization: vector maps. Software had been written $^{1,2,6}$ to process the bidirectional velocity maps into computer-generated vector cine maps to display the distribution of flow in the image plane (see figures). The individual vectors are displayed as tapered streaks with the thick end pointing in the direction of the flow and the length of the streak proportional to the relative velocity at the point of origin.

Data analysis. The angle between the long axis of the left ventricle and the ascending aorta was measured on the computer display of the coronal images or from hard copies. The maximum width of the aneurysms, the width of the aortic valvular ostium, and the ratio between these two widths were also measured (Fig. 1 and Table 1).

The cine vector maps were processed on an off-line computer and displayed there either in a cine format or individually.

\section{Results}

The results are summarized in Table II.

Normal subjects. Relatively consistent patterns of flow were seen in all cases in the ascending aorta, the arch, and the proximal descending aorta (distal descending aorta was not included in the imaged plane) with flow reversing predominantly at the transition of the arch and descending aorta. The flow was mostly axially directed in early systole with helicoid and retrograde secondary flow patterns developing through the latter half of systole and during diastole. Retrograde flow in late systole and in diastole was seen at the posterior left in the ascending aorta. Small vortices developed in the coronary sinuses predominantly during systole. The flow was therefore not truly laminar, not even in early systole, because it was not parabolic, and there were secondary and retrograde flows. For details see Kilner and asso- ciates. ${ }^{8}$ No vortices outside the coronary sinuses were present (Fig. 1).

Ascending aortic aneurysms (Marfan's syndrome)

Spin-echo imaging. The two patients with Marfan's syndrome had the typical finding of a greatly and symmetrically dilated ascending aorta, including the sinuses of Valsalva, ending abruptly at the origin of the innominate artery, giving rise to a shape that has been likened to a chemical retort or an Erlenmeyer flask (Fig. 2, $A$ ). The width of the aortic valvular ostium was increased in both cases (25 and $26 \mathrm{~mm}$ ) giving rise to moderate aortic regurgitation.

Velocity maps. At vector mapping, both patients had a central broad stream of blood minimally skewed to the right as it entered the dilated ascending aorta (Fig. 2, B). Such streams will be referred to as jets in the text from this point on. The reader should keep in mind that they are not narrow jets as in valvular stenosis, but look like jets as they enter a dilated vessel (aneurysm). The jet lasted until late systole. Already in early systole large vortices were formed on each side of the jet, clockwise on the left side and anticlockwise on the right side, as seen from the front, and larger in the right part of the aorta and in the right coronary sinus. The vortices lasted through systole and most of diastole and blood went around and around in the ascending aorta and the sinuses of Valsalva in diastole. In one of the patients, the vortices moved proximally during diastole. In the other patient two vortices on each side were formed, one in the proximal aorta including the sinus on each side and one on each side in the distal ascending aorta.

In one case, small vortices in the coronary sinuses appeared in early systole before the formation of the large vortices. These small vortices were similar to those seen in the normal subjects.

Retrograde flow from the arch joined the vortices more on the left side than on the right. Some of the retrograde flow crossed over from the left to the right sinus in early diastole (Fig. 2, C). Both patients had aortic valvular regurgitation and as the aortic valve opened retrograde flow in the ascending aorta was seen to meet antegrade flow at the aortic opening. Blood was seen to flow retrogradely from the great vessels into the aortic arch and down the ascending aorta as part of the aortic regurgitation.

Patient 2 also had severe thoracic scoliosis and a tortuous descending aorta that made two 90-degree bends. Signal loss caused by turbulence was seen at 
Table II. Summary of results

\begin{tabular}{|c|c|c|c|c|c|}
\hline $\begin{array}{l}\text { Anatomic } \\
\text { site }\end{array}$ & Normal & Marfan's syndrome & $\begin{array}{c}\text { Atherosclerotic ascending } \\
\text { aortic aneurysm }\end{array}$ & Dissection & Grafts \\
\hline $\begin{array}{l}\text { Sinuses of } \\
\text { Valsalva }\end{array}$ & Small systolic vortices & $\begin{array}{l}\text { Symmetric jet in early } \\
\text { systole. Large or small } \\
\text { vortices in two dilated } \\
\text { sinuses in continuity } \\
\text { with large vortices in } \\
\text { ascending aorta. }\end{array}$ & $\begin{array}{l}\text { Eccentric jet in systole. No } \\
\text { coronary sinus vortices } \\
\text { in systole, but the left } \\
\text { ascending aortic vortex } \\
\text { (see below) was feeding } \\
\text { the left coronary sinus } \\
\text { and coronary artery in } \\
\text { diastole. Separate vortex } \\
\text { in right coronary sinus } \\
\text { in diastole }\end{array}$ & & \\
\hline $\begin{array}{l}\text { Ascending } \\
\text { aorta }\end{array}$ & $\begin{array}{l}\text { Antegrade symmetric } \\
\text { flow in early and } \\
\text { mid systole. Retro- } \\
\text { grade flow in pos- } \\
\text { terior left stream in } \\
\text { mid to late systole } \\
\text { and diastole. }\end{array}$ & $\begin{array}{l}\text { Symmetric jet in early } \\
\text { systole. Two very } \\
\text { large expanding vorti- } \\
\text { ces in all of the as- } \\
\text { cending aorta. }\end{array}$ & $\begin{array}{l}\text { Eccentric jet in systole. } \\
\text { Very large vortex in the } \\
\text { anterior right moving } \\
\text { distally with flow all } \\
\text { through systole and di- } \\
\text { astole. Antegrade flow } \\
\text { along the right wall, ret- } \\
\text { rograde along the poste- } \\
\text { rior wall. }\end{array}$ & $\begin{array}{l}\text { Vortices starting } \\
\text { proximal to } \\
\text { intimal tear } \\
\text { and continu- } \\
\text { ing in the dis- } \\
\text { section. }\end{array}$ & $\begin{array}{l}\text { Highly disturbed flow } \\
\text { with one or several } \\
\text { proximal or distal } \\
\text { vortices. }\end{array}$ \\
\hline Aortic arch & $\begin{array}{l}\text { Forward symmetric } \\
\text { flow in most of sys- } \\
\text { tole. Retrograde } \\
\text { flow along the in- } \\
\text { ner curve in late } \\
\text { systole and dias- } \\
\text { tole. }\end{array}$ & Similar to normal. & Occasional vortices. & $\begin{array}{l}\text { Vortices starting } \\
\text { proximal to } \\
\text { intimal tear } \\
\text { and continu- } \\
\text { ing in the dis- } \\
\text { section. }\end{array}$ & $\begin{array}{l}\text { Highly disturbed flow } \\
\text { with one or several } \\
\text { proximal or distal } \\
\text { vortices. }\end{array}$ \\
\hline $\begin{array}{l}\text { Descending } \\
\text { aorta }\end{array}$ & $\begin{array}{l}\text { Antegrade symmetric } \\
\text { flow in most of sys- } \\
\text { tole. Retrograde } \\
\text { flow in anterior } \\
\text { stream in late sys- } \\
\text { tole and diastole. }\end{array}$ & Similar to normal. & Occasional vortices. & $\begin{array}{l}\text { Vortices starting } \\
\text { proximal to } \\
\text { intimal tear } \\
\text { and continu- } \\
\text { ing in the dis- } \\
\text { section. }\end{array}$ & $\begin{array}{l}\text { Highly disturbed flow } \\
\text { with one or several } \\
\text { proximal or distal } \\
\text { vortices. }\end{array}$ \\
\hline
\end{tabular}

the sharp bends in part of systole and irregular antegrade, retrograde, and transverse flows were seen in diastole.

\section{Atherosclerotic ascending aortic aneurysms}

Spin-echo imaging. Patients 3 through 5 (Table I) all had a differently shaped aneurysm compared with those of patients with Marfan's syndrome insofar as the aneurysm was confined to the anterior and right part of the ascending aorta, which had an asymmetric shape (Fig. 3, A). The sinuses of Valsalva were not involved in the aneurysms and the posterior and left wall of the ascending aorta appeared normal.

Velocity maps. The three first images representing approximately the first $150 \mathrm{msec}$ of the R-R interval showed only a large vortex in the aneurysm at the anterior right. No major stream forward or jet appeared until the fourth frame, and in all three patients the jet was eccentric and directed anteriorly and to the right into the aneurysm and hit its wall
(Fig. 3, B) (black in the vertical velocity map). Posterior to the jet and also anteriorly when the jet was no longer present in diastole was a large vortex that persisted through diastole and early systole (Fig. 3, C). The blood rotated counterclockwise as viewed from the front, and the vortex moved slightly in a distal direction through the cardiac cycle. The persistence of the vortex in diastole resulted in significant motion of blood in diastole in contrast to the finding in normal subjects in whom there was much less motion. All the forward flow was along the right wall and all retrograde flow along the posterior left wall. Mild aortic insufficiency was present in patients 3 and 5, but not in patient 4 . A small separate vortex was present in the right coronary sinus, and blood entered the left coronary sinus via the large vortex. In patient 4 , arrows were seen pointing toward the right coronary artery in systole and diastole and toward the left only in diastole. 

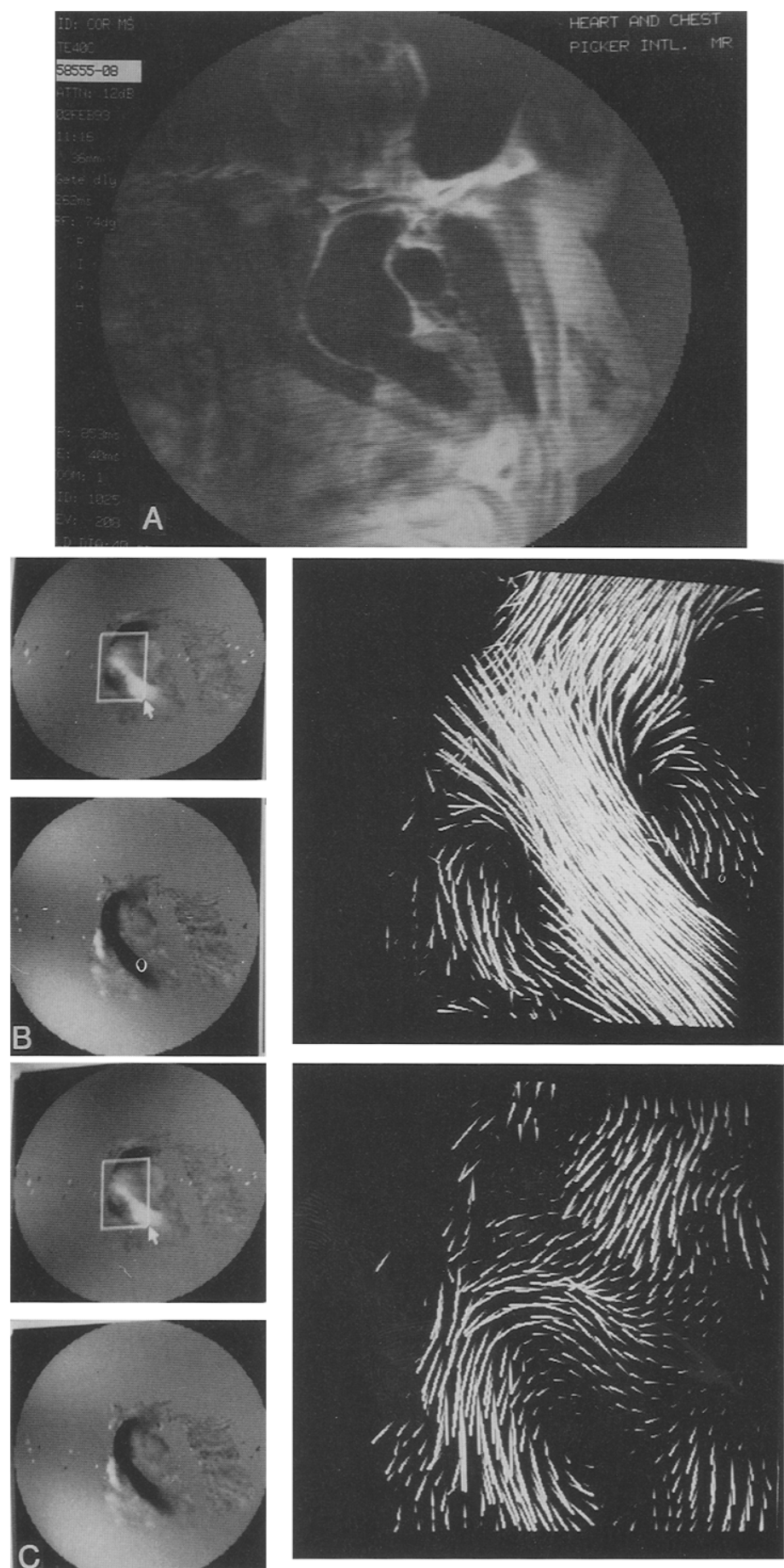

Fig. 2. Ascending aorta in patient with Marfan's syndrome (patient 2 in Table I). A, Spin-echo image in a coronal plane showing symmetric widening of the ascending aorta, including sinuses of Valsalva. Maximum widths of the aneurysm and aortic valve were measured, as well as the longitudinal axes of the left ventricle and the ascending aorta. B, Velocity and vector maps in oblique coronal plane equivalent to left anterior oblique projection in early systole with horizontal (upper left) and vertical (lower left) velocity encoding show broad symmetric jet and two large vortices in the sinuses of Valsalva. C, Same in middiastole showing retrograde flow to the left coronary sinus but also crossing over to the right above the sinuses into the right coronary sinus. In velocity images at the left, black represents velocity up the image in vertical encoding and to the right in the image in the horizontal encoding. White represents velocity down and left. The descending aorta is out of plane. 

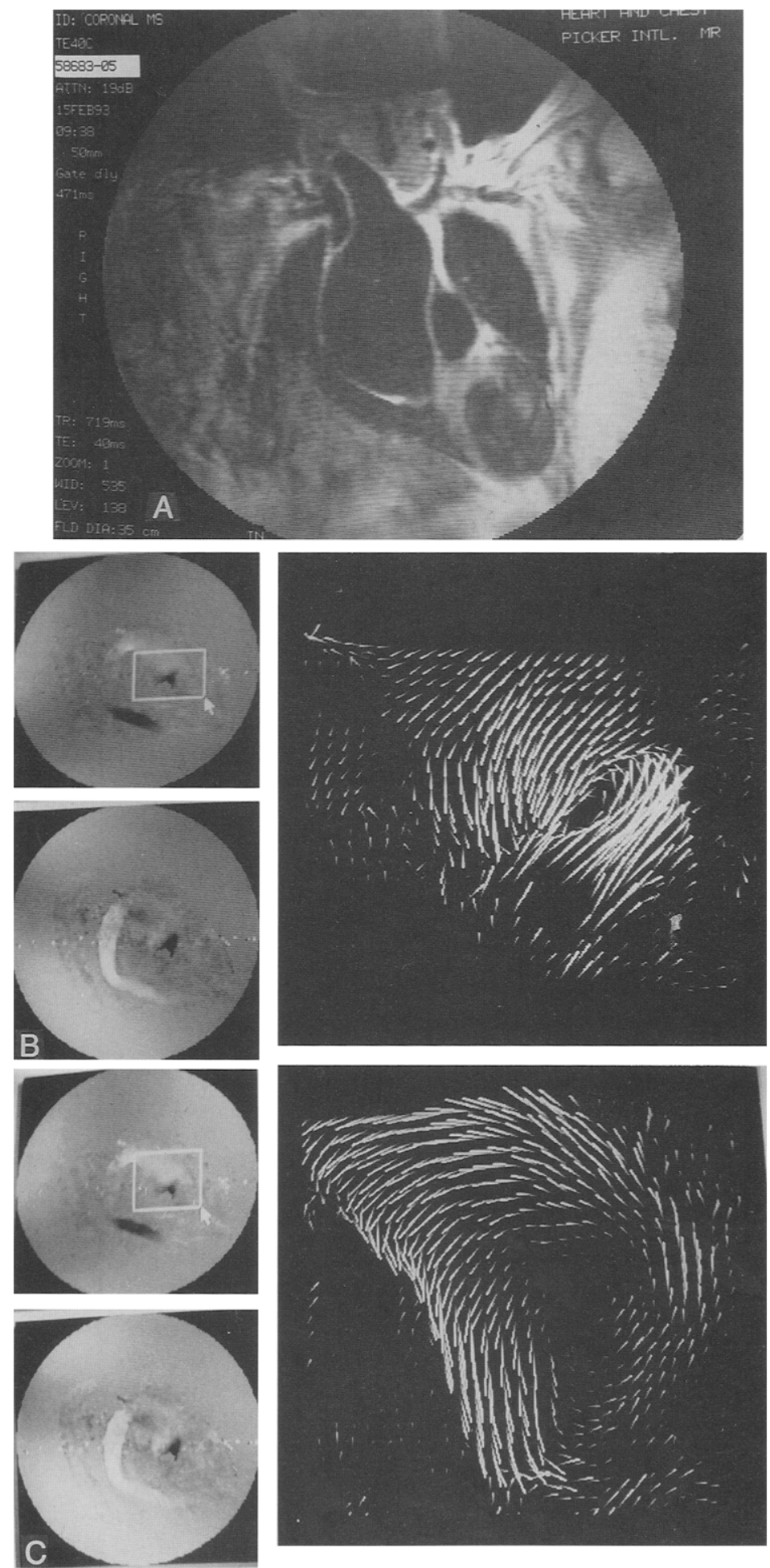

Fig. 3. Ascending aorta in a patient with atherosclerotic aneurysm (patient 3 in Table I). A, Spin-echo image in a coronal plane. The aneurysm is confined to the anterior right part of ascending aorta above the sinuses, and the posterior left part looks normal. B, Velocity and vector maps in an oblique coronal plane, equivalent to right posterior oblique projection in early systole with horizontal (upper left) and vertical (lower left) velocity encoding. The jet is eccentric (black in vertical velocity map) and hits the aneurysmal anterior left wall. $\mathbf{C}$, Same in early diastole shows a very large vortex in almost all of the ascending aorta. 

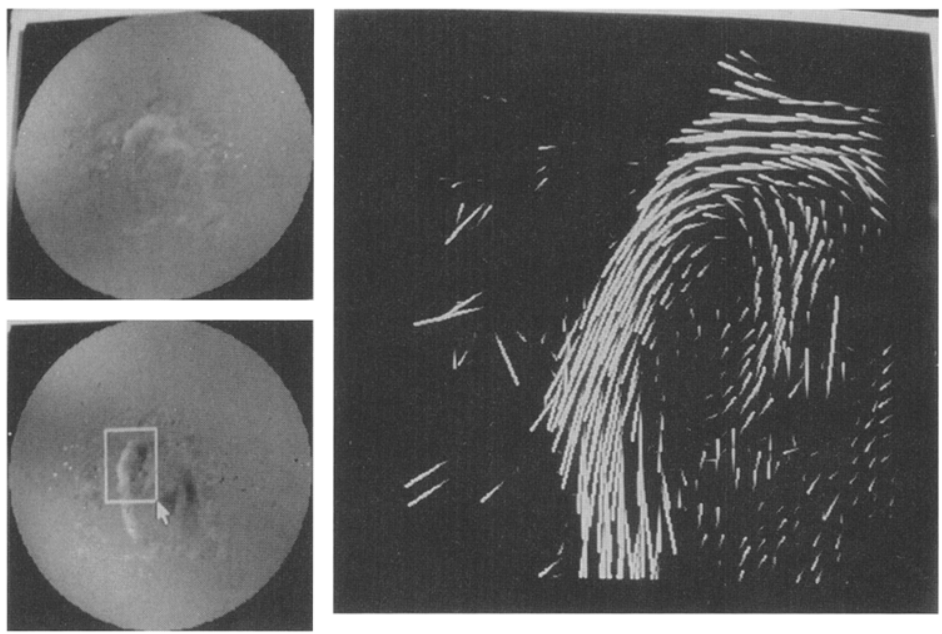

Fig. 4. Large vortex in descending aortic graft. Velocity maps with horizontal and vertical velocity encoding are to the left in the image. White represents velocity up and to the left in the image; black, the opposite.

The flow was most rapid along the inner curve of the arch in all three patients. A second vortex was created in the distal arch in patient 3 , who also had an aneurysmal proximal innominate artery with large inflow and vortex formation in the proximal arch. Patient 4 had an old dissection in the descending aorta in addition to the ascending aortic aneurysm. He had a vortex in the distal arch just proximal to the old dissection similar to that in patient 3 .

In Patient 5, flow reversal was seen in the proximal aorta, as well as in the middescending aorta. Patient 3 had a tortuous descending aorta (Fig. 3) with another vortex.

Ascending aortic aneurysm of unknown cause. The histologic finding in patient 6 was aneurysm, but there was no evidence of medionecrosis or other changes and no evidence of atherosclerosis or aortitis.

The anatomic and blood flow characteristics in this patient were almost exactly as in the patients with atherosclerotic aneurysm. Flow reversal was seen at the transition of the arch and descending aorta as in normal subjects, but there was additional flow reversal with vortex formation in the arch. There was a large amount of retrograde flow in the ascending aorta but no evidence of aortic regurgitation. There was also flow in various directions at various times in diastole in the arch.

Dissections. Three of the patients, 4, 7, and 8, had old dissections in addition to aneurysm or graft. There was disturbed blood flow in all dissections with vortices starting immediately proximal to the intimal tear and continuing in the true lumen of the dissection.

Grafts. There were four patients with grafts, including two with grafts in the ascending aorta after dissection several years earlier. Patient 8 , with a Dacron fabric graft, had a thrombosed false lumen in the arch and descending aorta, and Patient 7, with a homograft, has a separate dissection in the descending aorta. Patient 9 had a graft replacement of the aortic arch several years earlier because of an atherosclerotic aneurysm, and patient 10 had a graft in the descending aorta after trauma 4 months earlier. Both of these were Gelseal vascular grafts (Vascutek, Renfrenshire, Scotland).

Spin-echo images. The descending aortic graft was moderately dilated (width $31 \mathrm{~mm}$ compared with 18 $\mathrm{mm}$ in adjacent descending aorta) whereas the other grafts had normal or slightly increased width.

Velocity maps. Common to all grafts independent of position and age of the graft was that the blood flow was highly disturbed with vortices at both ends of the graft (arch), in the graft (descending aorta) (Fig. 4), and in the graft, as well as at the distal anastomosis (ascending aorta). There was a posterior jet in the dilated descending aortic graft and an anticlockwise vortex started as soon as blood entered the graft in systole (Fig. 4). The flow outside the grafts was also disturbed with other vortices and irregular flow not seen in normal subjects.

Miscellaneous arrow map findings. Not all of the coronary sinuses were included in the slices used for velocity mapping and all relevant flow was not seen 
in all cases. However, separate vortices in systole in the sinuses were seen in many cases. Retrograde flow at the posterior left in the ascending aorta into the left coronary sinus was seen, as well as flow crossing over above the left coronary sinus into the right sinus for right coronary artery diastolic flow (Fig. 2, C). Flow was also seen entering the sinuses from above and swirling around in them to continue as antegrade flow in the ascending aorta. The great vessels were not always included in the slices, but when one or two of them were, the following observations could be made: blood was seen to flow in and out of the great vessels from the arch, continuing retrogradely or sometimes antegradely in the arch. Arrow maps showed retrograde flow in the descending aorta going through the arch into the ascending aorta or going into the great vessels in diastole. Flow could sometimes be followed from a great vessel in diastole through the arch into the ascending aorta and sometimes into the descending aorta or another of the arch vessels.

There was more flow along the inner curve of the aortic arch in all observed cases.

Measurements. The angle between the longitudinal axis of the left ventricle and that of the aorta with the horizontal or transverse plane was measured in the patients and in 10 normal subjects. The angle averaged 30 degrees ( 25 and 35 degrees) in the patients with Marfan's syndrome, 45 degrees $(23,65$, and 47 degrees $)$ in the patients with atherosclerosis, and 21 degrees ( 15 to 26 degrees) in the 10 normal subjects. It was an average of 30 degrees in all the 10 patients.

The ratio between the maximal aneurysmal width and the width of the aortic valve was 2.55 (2.5 and 2.6) in the patients with Marfan's syndrome and 3.4 (3.2 to 3.7 ) in the three patients with atherosclerotic aneurysms. The difference was because of widening of the aortic valve in the patients with Marfan's syndrome (25.5 versus $21 \mathrm{~mm}$ average) inasmuch as the width of the aneurysm was not significantly different (66 versus $69 \mathrm{~mm}$ ).

\section{Discussion}

Morphologic features of ascending aortic aneurysms. The two Marfan's syndrome aneurysms had the classic appearance of a pear-shaped symmetric dilation confined to the ascending aorta and including the sinuses of Valsalva, which is well known from x-ray angiography, echocardiography, ${ }^{9}$ computed tomography, ${ }^{10}$ and earlier MRI studies. ${ }^{11-16} \mathrm{We}$ also reviewed the spin-echo images in two other patients with Marfan's syndrome who did not have vector mapping. The shape of the ascending aorta was identical to that of the others.

The three atherosclerotic ascending aneurysms and two others that were not subjected to velocity mapping had also a characteristics shape. In all five patients, the aneurysms were confined to the anterior right part of the ascending aorta and the posterior left part of the sinuses appeared normal (see Fig. 3, $A$ ). This is in agreement with our and others' experience from x-ray aortograms, in which the aneurysms are more prominent on the right, the outside, or the convex side of the ascending aorta on left anterior oblique projections. However, we are not aware that this shape, seen in Fig. 3, $A$, has ever been described in detail in the radiologic, surgical, or pathologic literature. ${ }^{17.19}$ This is probably because MRI can image morphologic features in more detail than other techniques, including autopsy and specimen studies, so that the aneurysmal lumen, its walls, and all the adjacent structures are seen simultaneously. The morphology of these five atherosclerotic ascending aortic aneurysms is quite diagnostic and sets them apart from the Marfan's syndrome aneurysms (Fig. 2, $A$ ) although probably not from inflammatory aneurysms (see following section).

Atherosclerotic aneurysms are rare in the ascending aorta and much more common distally. ${ }^{18}$ The most common cause of aneurysms in the ascending aorta is degenerative disease of the media (medionecrosis and mucoid degeneration) as seen in Marfan's syndrome or forme fruste of Marfan. Inflammatory diseases predominate in the arch and descending aorta in our time, whereas they were the most common, and most often appeared in the ascending aorta, when syphilis was the dominant cause of aneurysms. ${ }^{17}$ We have no experience with MRI of luetic or other inflammatory aneurysms, but they are described in the pathologic literature as being anterior, ${ }^{17}$ probably similar in location to atherosclerotic aneurysms.

The ascending aortic aneurysm with normal or uninterpretable histologic findings (case 6, Table I) looked exactly like the atherosclerotic aneurysms. The patient was only 39 years old, which makes atherosclerosis unlikely. We assume by exclusion that this aneurysm was inflammatory.

Blood flow patterns in ascending aortic aneurysms. The main difference between the atherosclerotic and the Marfan's syndrome aneurysms was that the jet was obliquely directed toward the right anterior wall in the atherosclerotic aneurysms and was central in Marfan's syndrome and that one large vortex to the left of the jet formed in the former 
whereas there were two large vortices, one on each side of the jet, in the latter (see Results section and Figs. 2 and 3). This is probably to be expected from the morphologic features of the aneurysms, but it could also be the other way around, that is, that the morphologic features are a result of hydraulic forces exerted by the jet or the vortices, or both forces. A possible cause of an eccentric jet would be misalignment between the left ventricle and the ascending aorta; in other words, that a more horizontally aligned ventricle would eject blood to the right into a more vertically directed aorta. We therefore measured the inclination of the left ventricular long axis and that of the ascending aorta with the transverse or horizontal plane in the patients and compared the results with those in 10 normal subjects. The left ventricle was more horizontally oriented than the aorta in all cases, but there was no significant difference between the results in the normal subjects and those in the patients with Marfan's syndrome or atherosclerotic aneurysm in this small series. The angle was quite large in two patients with atherosclerotic aneurysms (65 and 47 degrees) but normal in one (23 degrees), and it was very small (9 degrees) in the patient with an unclear aneurysmal cause but with an aneurysm of the same morphologic type as the atherosclerotic aneurysms. It seems therefore unlikely that the apparent misalignment would exert much effect on the morphologic features of ascending aortic aneurysms. It may seem difficult to explain this apparent misalignment between the left ventricle and the aorta, which is also seen in normal subjects, but one must keep in mind that the records are two-dimensional and that a twisting motion of the left ventricle may align the left ventricle and aorta, at least in normal subjects.

We also looked into the possibility that the relationship between the width of the aortic valvular ostium and the aneurysm might be different and possibly play a role in the formation or hemodynamics of the two types of ascending aortic aneurysms (see Table I and Results section). The ratios were smaller in the patients with Marfan's syndrome, most likely because of a stretched aortic ring, which increased the numerator. Both patients had aortic insufficiency. One patient with atherosclerotic aneurysm had also a wide aortic valvular ostium with a 25 mm diameter, like the patients with Marfan's syndrome, but had no aortic regurgitation.

Basic fluid mechanical principles would suggest the jet to be central in aneurysms, ${ }^{20}$ as it indeed is in
Marfan's syndrome. Computer simulation of blood flow patterns on the basis of solving continuity and Navier-Stokes equations in a fusiform aneurysm also suggested that the jet should be central. ${ }^{21}$ The severely asymmetric jet in atherosclerotic aneurysms is therefore difficult to explain.

The fact that the jet hits the wall of the atherosclerotic aneurysm may suggest that it exerts an influence on the further expansion of the aneurysm. On the other hand, such a jet is missing in the symmetric Marfan aneurysms, which attain the same width without a jet hitting any wall. The vortices in the expanding parts of both types of aneurysms cannot be excluded to play a role in their further growth, although the hydrodynamic force is likely to be much smaller than the tension force. According to the law of La Place, the wall tension (for a given blood pressure) increases in proportion to the square of the radius of the curvature. ${ }^{22}$ Where diameter increase is large, this has major consequences for tension in a wall that is (presumably) already structurally abnormal. The more the vessel dilates, the more it is subjected to stresses that predispose to further dilation. For a given flow rate, mean forward velocity decreases in inverse proportion to the square of the diameter. When flow moves from a region of normal diameter into a region of increased diameter, momentum is conserved, and the stream maintains its initial velocity, but separates from the diverging wall or walls. ${ }^{20,21}$ Flow separation is associated with recirculation, that is, local reversal of flow, in the separation zone. The recirculating movement can take the form of a vortex. The pulsatility of flow and any asymmetry or dilation can enhance the tendency to vortex formation.

It is therefore likely that aneurysm formation is primary and dominant and has fluid dynamic consequences that are secondary and do not contribute significantly to the propagation of the aneurysms.

The vortices sometimes moved distally in systole, there were sometimes multiple vortices, and there was, in general, irregular flow, not only in the aneurysms, but also in the arch with recirculations not seen in normal subjects. ${ }^{8,23}$ The blood flow was therefore quite disturbed, which may influence flow to the whole body, perhaps especially to the coronary arteries and brain, which depend on a finetuned interplay between antegrade and retrograde flow. ${ }^{23-26}$

Blood flow in dissections. The three dissections that were unrelated to the aneurysms or grafts all 
had disturbed blood flow with vortices. Patient 4 had an atherosclerotic ascending aortic aneurysm, a relatively normal arch without aneurysm, and a separate dissection in the descending aorta. The hemodynamic trauma appeared to be in the right anterior wall of the ascending aorta, which was hit by the jet, and one would expect the dissection to start there, if there was much force in a jet. The dissection in the descending aorta seems to support our view that morphologic changes are primary and not the result of hydraulic, hemodynamic forces.

Blood flow in grafts. Common to all grafts was a rather severely disturbed blood flow with formation of single or multiple vortices. This was the case also in the graft that was only 3.5 months old (see Results section and Fig. 4). There were vortices in and around the grafts and also disturbed flow elsewhere, suggesting a nonideal situation. Approximately 200,000 prosthetic vascular grafts are implanted per year in the United States, and there has been and is much ongoing research to find the ideal graft, which has been defined as follows: "For a synthetic vascular graft to function as a normal blood vessel, it must contain some or all of the activities and properties which make natural blood vessels function normally. These include prostacyclin $\left(\mathrm{PGI}_{2}\right)$, endotheliumderived relaxing factor (EDRF), tissue plasminogen activator (tPA), heparin and other glycosaminoglycans, thrombomodulin, ADPase, compliance, and undoubtedly other as yet unknown factors." ${ }^{.7}$ Emphasis has been placed on the factors mentioned, ${ }^{28}$ but we have not had a tool until now to examine how the grafts carry blood flow. This study of blood flow patterns in grafts is small, but suggests that further research be done regarding blood flow in grafts of varying age and composition, anastomosis technique, and so on. Magnetic resonance velocity mapping is a powerful noninvasive tool to be used in research regarding the ideal prosthetic vascular graft, which, with due respect for all the aforementioned activities and properties, first and foremost must carry blood flow in a reasonably normal fashion.

Miscellaneous blood flow observations. Blood was seen to enter the left coronary sinus from above in diastole and also to cross over from the left to the right just above the sinuses (Fig. 2, $C$ ) as predicted from earlier indirect studies that used velocity mapping in the mid ascending aorta. ${ }^{24}$

Blood was also seen to flow in and out of the great vessels in the arch in various ways as described in detail in the Results section, which was likewise predicted in earlier studies that used through-plane one-directional velocity mapping. ${ }^{23,26}$ Multidirectional MRI velocity imaging with computer-generated vector maps is an ideal tool to study flow patterns in the aorta and its major branches.

Limitations of the study. The main limitation of the flow pattern analysis is that it was confined to a $10 \mathrm{~mm}$ slice of the aorta and that only two directions were recorded. It is possible to make a complete "seven-dimensional" analysis of the flow, ${ }^{29}$ but it would be enormously time consuming and not practical at the present speed of MRI studies. The anterior-posterior direction was not analyzed in the patients and it is quite possible, even likely, that more vortices are present in that direction.

The study is also limited in the numbers of the different categories of aneurysms and grafts, but shows features of disturbed flow common to all categories.

We did not analyze the consequences of the disturbed flow, but because it involves energy losses it must increase cardiac work. Neither did we have the opportunity to analyze flow during exercise.

Conclusions. MRI spin-echo imaging and multidirectional cine velocity mapping with computergenerated velocity vectors are suitable noninvasive techniques to reveal detailed morphologic features and blood flow patterns in thoracic aortic aneurysms and grafts. All aneurysms, grafts, and dissections had severely disturbed blood flow, which entails energy losses and must increase cardiac work. The technique may be applied to test efficiency of grafts.

\section{REFERENCES}

1. Yang GZ, Burger P, Mohiaddin RH. In vivo blood flow analysis and animation for magnetic resonance imaging. Proc Int Soc Opt Engineering (SPIIE) 1990; 1233:176-82.

2. Mohiaddin RH, Yang GZ, Burger P, Firmin DN, Longmore DB. Automatic enhancement, animation, and segmentation of flow in the peripheral arteries from magnetic resonance phase shift velocity mapping. J Comput Assist Tomogr 1992;16:176-81.

3. Napel S, Lee DH, Fryane R, Rutt BK. Visualizing three-dimensional flow with simulated streamlines and three-dimensional phase contrast MR imaging. J Magn Reson Imaging 1992;2:143-53.

4. Walker PG, Cranney GB, Scheidegger MB, Waseleski G, Pohost GM, Yoganathan AP. Computer animation of the time dependent flow field in a human left ventricle: an in vivo NMR phase velocity 
encoding study [Abstract]. Soc Magn Reson Med 1992;2:2515.

5. Yang GZ, Burger P, Kilner PJ, Mohiaddin RH. In vivo blood flow visualization with magnetic resonance imaging. IEEE Proceedings of Visualization, San Diego, Calif. 1991:202-9.

6. Buonocore MH. Algorithms for improving streamlines in 3-D phase contrast angiography. Magn Reson Med 1994;31:22-30.

7. Mohiaddin RH, Yang GZ, Kilner PJ, Longmore DB. Visualization of flow by vector analysis of multidirectional cine magnetic resonance velocity mapping: technique and application. J Comput Assist Tomogr 1994;18:383-92.

8. Kilner PJ, Yang GZ, Mohiaddin RH, Firmin DN, Longmore DB. Helical and retrograde secondary flow patterns in the aortic arch studied by three-directional magnetic resonance velocity mapping. Circulation 1993;88(Pt 1):2235-47.

9. De Maria AN, Bommer W, Neumann A, Weinert L, Bogren HG. Identification and localization of aneurysms of the ascending aorta by cross-sectional echocardiography. Circulation 1979;59:755-61.

10. Dinsmore TE, Liberthson RR, Wismer GL, et al. Magnetic resonance imaging of thoracic aortic aneurysms: comparison with other imaging methods. AJR Am J Roentgenol 1986;146:309-14.

11. Amparo EG, Higgins CB, Hoddick W, et al. Magnetic resonance imaging in aortic disease: preliminary results. AJR Am J Roentgenol 1984;143:1203-9.

12. Boxer RA, La Corte MA, Singh S, Davis J, Goldman M, Stein HL. Evaluation of the aorta in the Marfan syndrome by magnetic resonance imaging. Am Heart J 1986;111:1001-2.

13. Schaefer S, Peshock RM, Malloy CR, Katz J, Parkey RW, Willerson JT. Nuclear magnetic resonance imaging in Marfan's syndrome. J Am Coll Cardiol 1987;9:70-4.

14. Soulen RL, Rishman EK, Pyeritz RE, Zerhouni EA, Pessar ML. Marfan syndrome: evaluation with MR imaging versus CT. Radiology 1987;165:697-701.

15. Spielmann RP, Sehlz B, Schofer J, Witte G, Heller M. Kernspintomographie des thoracalen Aortenaneurysmas. Fortschr Rontgenstr 1988;149:571-5.

16. Grenier P, Pernes JM, Desbleds MT, DeBrux JL. Magnetic resonance imaging of aneurysms and chronic dissections of the thoracic aorta. Ann Vasc Surg 1987;1:534-41.
17. Boyd LJ. Aneurysm of the thoracic aorta. Am J Med Sci 1924;168:654-68.

18. Leu HJ, Jülke M. Das thoracale Aortenaneurysma: pathologisch anatomische Analyse von 111 Fällen. Schweiz Med Wochenschr 1984;114:1593-5.

19. Doerr W. Thoracic aortic aneurysms. Thorac Cardiovasc Surg 1987;35:111-21.

20. Goldsmith HL, Karino T. Flow and vascular geometry. In: Hwang NHC, Tutto VT, Yen MRT, eds. Advances in cardiovascular engineering. New York and London: Plenum Press, 1992:127-50.

21. Wong PKC, Johnston KW, Ethier CR, Cobbold RSC. Computer simulation of blood flow patterns in arteries of various geometries. J Vasc Surg 1991;14:658-67.

22. Fung YC. The physical principles of circulation. In: Fung YC, ed. Biodynamics of the circulation. New York: Springer Verlag, 1984:1-21.

23. Bogren HG, Buonocore MH. Blood flow measurements in the aorta and major arteries with magnetic resonance velocity mapping. J Magn Reson Imaging 1994;4:119-30.

24. Bogren HG, Klipstein RH, Firmin DN, et al. Quantitation of ante and retrograde blood flow in the human aorta by magnetic resonance velocity mapping. Am Heart J 1989;117:1214-22.

25. Bogren HG, Mohiaddin RH, Slipstein RH, et al. The function of the aorta in ischemic heart disease: a magnetic resonance and angiographic study of aortic compliance and blood flow patterns. Am Heart J 1989;118:990-9.

26. Bogren HG, Buonocore MH, Gu W-Z. Carotid and vertebral artery blood flow measurements by MR velocity mapping in left and right-handed normal subjects. J Magn Reson Imaging 1994;4:37-46.

27. Didisheim P. Current status of biomaterials: use and development. In: Hwang NHC, Tutto VT, Yen MRT, eds. Advances in cardiovascular engineering. New York and London: Plenum Press, 1992:189-95.

28. Phifer TJ, Hwang NHC. Vascular grafts: clinical and hemodynamic applications. In: Hwang NHC, Tutto VT, Yen MRT, eds. Advances in cardiovascular engineering. New York and London: Plenum Press, 1992:385-400.

29. Firmin DN, Gatehouse PD, Yang GZ, Kilner PJ, Longmore DB. Rapid 7-dimensional imaging of pulsatile flow [Abstract]. Soc Magn Reson Med 1992;11: 212. 